\section{CHILD PSYCHIATRY: A PAEDIATRIC PERSPECTIVE OF PSYCHO-SOCIO-CULTURAL ISSUES IN PAKISTAN}

Nighat Jahan Nadeem*, Muhammad Shoaib Sulaiman. South West London and St George's Mental Health NHS Trust, UK

10.1136/archdischild-2021-europaediatrics.37

Deeply entrenched socio-cultural norms dictate everyday life In Pakistan and it is vital that correct attitudes towards Child and Adolescent Mental Health (CAMH) are fostered. In a sub-specialist field where early diagnosis is imperative in securing the best possible outcome, its importance cannot be overemphasised and is of public interest. Despite having potentially life-long consequences, there is inadequate awareness, scarce CAMH facilities and a significant dearth of research about CAMH in Pakistan. This study aims to explore the views of doctors and students towards Paediatric Psychiatry in Pakistan.

Doctors were recruited by convenience sampling and a snowballing method was used to recruit participants. They were asked to complete an online survey and data was analysed both quantitatively and qualitatively. Approval was granted by the Research \& Development Department at South West London and St George's Mental Health NHS Trust, UK.

There were 80 participants; the vast majority $50(62.5 \%)$ were Paediatric doctors, 30(37.5\%) were other specialists/student doctors. $85 \%$ of all participants felt that increasing the number of child psychiatry specialists would be beneficial and all participants felt that the main issues facing CAMH in Pakistan are related to parents, infrastructure and taboos/socio-cultural stigma.

Of the 50 paediatric doctors, 30/50(60\%) had <10years experience in Paediatrics. Identifying and referring to mental health specialists was the most common role of paediatric doctors $32 / 50(64 \%)$ in CAMH cases whereas

$15 / 50(30 \%)$ reported that they are involved in initiating psychotropic treatment. Paediatric doctors felt less competent in prescribing psychotropic medication in children than in counselling parents where they felt more competent: 46/50 $(92 \%)$ of paediatric doctors reported being 'little/not at all' competent in prescribing psychotropic medication to children whereas 47/50(94\%) reported feeling 'little/very' competent in counselling parents about CAMH issues.

Taboo and socio-cultural stigma form a large barrier in $\mathrm{CAMH}$ in Pakistan and a specific anti-stigma campaign is needed to lift the veil of socio-cultural taboos, broaden the nation's vision and gain a clear perspective.

The way forward requires multidisciplinary collaboration to educate, empower and engage all levels of society. Improving infrastructure, living environment and economic stability as well as promoting public and professional education is vital. Media campaigns involving celebrities/influential figures and leaders (political and religious) need to be disseminated to bring Paediatric Psychiatry into the limelight.

Increased emphasis on identifying and managing $\mathrm{CAMH}$ disorders will help shift socio-cultural norms to remove the shackles of stigma and relieve the silenced agony.

\section{GUIDELINES FOR ACUTE OTITIS MEDIA IN CHILDREN WORLDWIDE: USEFUL OR USELESS?}

Elena-Lia Spoială*, Eduard Roşu, Cristian Duşa, Cristina Gavrilovici. Universitatea de Medicină şi Farmacie, Grigore T.Popa' laşi, România

10.1136/archdischild-2021-europaediatrics.38
Goal Our aim is to identify, analyze and compare the international guidelines or national consensus reports on the management of acute otitis media (AOM) in children.

Method We performed a systematic search on PubMed database using the keywords: 'acute otitis media' AND ['children' OR 'pediatric' OR 'paediatric'] AND ['guideline' OR 'consensus']. We excluded publications in other languages than English or French and animal studies. A total of 249 papers have been initially found. After exclusion of those that were not relevant (reviews, case series, case reports) we ended up in gathering 27 publications. After full text screening, we excluded papers that did not specifically refer to AOM diagnosis and management. Finally 13 papers matched the criteria. Results We found out 11 countries having specific AOM guidelines published in Pubmed: USA, Spain, France, Germany, South Africa, Australia, Korea, Canada, Italy, UK and Japan. Prompt antibiotic treatment is recommended for: 1. all children with temperature over 39 gr C or with otorrhea (Germany, Italy), 2. all children withintracranial complications and/ or a history of recurrence (Italy, South African), 3. all children under the age of 2 years (Italy, South Africa, France), 4. children over 2 years old with severe bilateral AOM (Italy), or with failure to respond to symptomatic treatment after 48 to 72 hours (Australia), 5. all children with signs of severe infection (temperature $>38$, othorea, otolagia $>48$ hours) (South Africa, France), 6. children with risk factors: malnourished, immunodepression, ear malformation) (France and South Africa), day-care attendees or siblings of children attending day care centers (South Africa). Amoxicillin is universally accepted as the first-line antibiotic therapy in all included guidelines. The alternative for children allergic to penicillins is the use of macrolides. Some of the guidelines mention the importance of preventive strategies (Italy, Germany, Australia, USA, Spain, Japan, Korea, South Africa). Besides reducing the risk factors, almost all the guidelines encourage pneumococcal and influenza vaccination. The exception is the Spanish guideline which refers to previous studies that showed no direct beneficial effect after vaccination.

Conclusion These guidelines revealed similarities in many aspects, with variations in specific recommendations, due to local epidemiology and follow-up possibilities. In order to reduce the healthcare burden, there is a special need for developing a global guideline with specific recommendations according to epidemiological differences.

\section{NOTIONS, KNOWLEDGE, AND PERCEPTIONS OF PAIN RELATED TO MINOR PAINFUL PROCEDURES AMONG MEMBERS OF A PEDIATRIC DIVISION}

María José Conejero*, Camila Carril, Marcela Ortiz, Trinidad Pardo, Leonor Martínez. Unicersidad Católica de Chile

\subsection{6/archdischild-2021-europaediatrics.39}

Explore notions, knowledge, and perceptions of members of a pediatric division of a Chilean University towards pain related to minor procedures (MPP), mainly venous punctures and vaccination.

An online survey was designed and sent via email to all members of a Pediatric Division between May and June 2019. The questionnaire explores technical knowledge and experience in managing pain related to MPP as well as barriers 
perceived in the implementation of successful mitigation strategies. Data was later utilized for descriptive analysis.

The survey was sent to 317 participants, obtaining 71 answers (22,3\% response rate). $23 \%$ of the respondents where pediatric residents. $84,5 \%$ consider that managing pain related to MPP is relevant and must be addressed in every patient. The procedures perceived as most painful (scale from 1 to

10) were arterial punctures (median 9), followed by lumbar punctures and venous punctures (median 7 both). The entire sample declared knowing at least one measure to reduce pain related to MPP, where the most common were: distraction $(84,5 \%)$ non-nutritious suctioning $(78,9 \%)$ and topical creams (76\%). Still, $15,5 \%$ of respondents routinely do not use any measure. $40 \%$ declare that during their training they were taught that pain related to MPP should always be managed in a multimodal way, existing effective measures available. 94,4\% of respondents believe that there are consequences regarding this type of pain; yet, half of the respondents agree that consequences are minimal and last only for a short period of time. The main barriers against implementation identified are a lack of knowledge of existing available measures $(78,9 \%)$ and a belief that these require additional time (60,6\%).

There is concern among respondents regarding the need for adequate management of this type of pain. In spite of this, a large proportion of participants do not routinely use any measure. This might be explained by an underestimation of the important consequences of this type of pain. The low response rate obtained is one of the main limitations of the study. The findings of this survey could assist in planning better and more effective strategies to support the use of effective measures to reduce pain related to MPP among pediatricians.

\section{OSTEOMYELITIS MIMICKING AS NON-ACCIDENTAL INJURY IN EX PREMATURE INFANT}

A Kaninde*, M Wayland, M Chomicki, R Ramaswamy. Peterborough City Hospital, UK

10.1136/archdischild-2021-europaediatrics.40

Introduction Non-Accidental injury (NAI) in children is an important cause of major injury, morbidity and mortality in children. Often times posing as diagnostic challenge to differentiate from underlying pathological cause[1-3]. The Trauma Audit Research Network (TARN) report 'Severe injury in Children 2012', identified that children under the age of 2 years who suffered significant trauma, were found to have NAI as the root cause. Out of those, the majority of cases with suspected NAI $(76 \%)$ were less than 1 year of age[4]

Case Summary We report a case of 33 weeks to immigrant family, presenting to Accident and Emergency department at 7 weeks of age with painful movement of left shoulder. Mother gave history of hearing a 'pop' while changing his vest on day of presentation. Due to language barrier, there was delay in seeking medical help.

Due to delay in seeking help and implausible mechanism of injury, a suspicion of non-accidental Injury was raised and a child protection medical examination was performed.

On examination, it was noted that he had painful movements of left shoulder joint with asymmetrical Moro's reflex. There were no other physical injury marks or bruises.
$\mathrm{X}$ ray of left shoulder was reported as indicative of possible periosteal elevation of left mid-shaft humerus with a recommendation to consider further imaging.

On second day of admission, patient had low grade pyrexia prompting septic screen. On further imaging, MRI shoulder was performed revealing changes suggestive of osteomyelitis.

His initial inflammatory markers were normal. Diagnosis of osteomyelitis was confirmed based on temperature spikes, MRI finding and clinical improvement in range of movements in left arm after starting on intravenous antibiotics.

Discussion Considering a diverse spectrum of presentation for child abuse, it can throw diagnostic challenges to any clinician to differentiate between a pathological cause and Non accidental Injury[3].We had to consider brachial plexus injury, Cerebral palsy as differentials as patient was afebrile initially. It demonstrates how close; range of differentials could be. In past, there are anecdotal example of Neonatal osteomyelitis presenting as Non accidental injury[5].

Prematurity, socio- economic background are independent risk factors contributing to NAI[6]. In one of the American studies, they described NAI doubles risk of mortality by twofold [7], so one should be very vigilant in dealing with cases of suspected NAI

Conclusion Our case highlights importance of non-judgemental and neutral approach while dealing with cases of suspected NAI.

\section{RISK OF OBESITY AND INFANT FEEDING PRACTICES: RESULTS OF PARENTAL SURVEY}

Vitalii Kukushkin*, Olena Starets, Natalia Kotova. Odesa National Medical University

10.1136/archdischild-2021-europaediatrics.41

Overweight and obesity in children seem to be quite common problem in many countries. The food dominants and preferences are developing in early childhood. Not rationale feeding of infants and their overweight are risk factors for obesity in school age. The goal of study was to assess the physical development and feeding practice of infants based on self reported parental survey.

The anonymous parental survey for parameters of physical development and feeding practice details was done randomly. The parents of 2 nd - 3rd year old children were asked to complete special Google-forms. The individual data, overall tables and diagrams were obtained on-line.

Totally 168 filled questionnaires were processed. The data for body weight in the age of 1 year (z-score) shown that $22,6 \%$ children had weight $>+2 \sigma ; 1,8 \%$ children had weight $>+3 \sigma$. For body length it was revealed that $8,9 \%$ children had parameter $>+3 \sigma$. The weight/length indicator for $8,9 \%$ children was $>+2 \sigma$; for $3,0 \%$ children $>+3 \sigma$. The natural feeding since birth was started for $88,1 \%$ of children; until 6 months $61,9 \%$ of children received breast milk, until 12 months $-54,8 \%$. From those children who received breast milk 35,1\% were given also a water during first months. The practice of scheduled feeding followed in 19,1\% families. The first solid food in the age of 4-6 months was administered in $91,6 \%$ of children; mostly presented by vegetable pure. Part $(49,6 \%)$ of children received a meat since the age of 7 months. In the age of 9-12 months feeding of infants was quite diverse. But many parents gave to babies food or drinks 